Pacific

Journal of

Mathematics

\title{
SINGULAR FIBERS AND 4-DIMENSIONAL COBORDISM GROUP
}

\author{
OSAMU SAEKI
}




\title{
SINGULAR FIBERS AND 4-DIMENSIONAL COBORDISM GROUP
}

\author{
OSAMU SAEKI
}

\begin{abstract}
Using the technique of singular fibers of $C^{\infty}$ stable maps, we give a new proof to the theorem, originally due to Rohlin, that the oriented cobordism group of 4-dimensional manifolds is infinite cyclic and is generated by the cobordism class of the complex projective plane. A byproduct is a new and transparent proof of the signature formula, originally due to $T$. Yamamoto and the author, for 4-dimensional manifolds in terms of singular fibers.
\end{abstract}

\section{Introduction}

Saeki [2004] developed the theory of singular fibers of generic differentiable maps $f: M \rightarrow N$ between manifolds with $\operatorname{dim} M>\operatorname{dim} N$. In particular, $C^{\infty}$ stable maps with $(\operatorname{dim} M, \operatorname{dim} N)=(2,1),(3,2)$ and $(4,3)$ were thoroughly studied and their singular fibers were completely classified up to a natural equivalence. (For precise definitions, see [Golubitsky and Guillemin 1973] and Section 2. For the case of maps between nonorientable manifolds, see [Yamamoto 2006] as well.)

In this paper, we use these classifications of singular fibers to determine the structures of $\mathfrak{N}_{2}, \Omega_{2}, \Omega_{3}$ and $\Omega_{4}$, where $\mathfrak{N}_{n}$ and $\Omega_{n}$ are the cobordism group and oriented cobordism group, respectively, of manifolds of dimension $n$. These groups were central objects of study in differential topology in the middle of 20th century, and their structures have been completely clarified. Our main objective here is to use classifications of singular fibers of $C^{\infty}$ stable maps to show that $\Omega_{4}$ is an infinite cyclic group generated by the cobordism class of the complex projective plane, and that the signature function $\Omega_{4} \rightarrow \mathbb{Z}$ gives an isomorphism. This theorem is originally due to Rohlin [1952]; see also [Guillou and Marin 1986].

The idea of our proof, which is constructive, is as follows. If we have a smooth map $f$ of a closed oriented 4-manifold $M$ into a 3-manifold $N$, then a regular fiber is a finite disjoint union of circles. In particular, if $f$ is nonsingular, then $M$ is a circle bundle over a 3-manifold and therefore bounds the associated 2-disk bundle.

MSC2000: primary 57R45; secondary 57R75.

Keywords: singular fiber, stable map, cobordism group, signature, complex projective plane. This work was supported in part by Grant-in-Aid for Scientific Research (B) Number 19340018, Japan Society for the Promotion of Science. 
If $f$ has singularities and is generic enough, then the 4-manifold $M$ is decomposed into several pieces according to the classification of singular fibers. For example, regular fibers correspond to a circle bundle over a 3-manifold. Furthermore, we can find a "canonical" 5-manifold for each such piece, except for that corresponding to specific singular fibers, called singular fibers of type III $^{8}$ in [Saeki 2004]. By gluing these 5-manifold pieces to $M \times[0,1]$, we get a cobordism between $M$ and a finite disjoint union of copies of a certain 4-manifold, each corresponding to a singular fiber of type III $^{8}$. In this paper, we will show that this 4 -manifold is in fact diffeomorphic to the complex projective plane $\mathbb{C} P^{2}$ up to orientation. This observation shows that $\mathbb{C} P^{2}$ is a natural representative of the generator of the 4-dimensional oriented cobordism group $\Omega_{4}$, since our proof is natural and the appearance of $\mathbb{C} P^{2}$ is not artificial. We may also say that we give a modern proof of the classical Rohlin's theorem from a singularity theoretical viewpoint.

As a corollary to our argument, we get a new and constructive proof of the signature formula proved in [Saeki and Yamamoto 2006]: the signature of a 4manifold $M$ coincides with the number of singular fibers of type III $^{8}$ counted with signs for any $C^{\infty}$ stable map $f: M \rightarrow N$ into a 3-manifold. The proof depended on the classification of singular fibers of $C^{\infty}$ stable maps of $n$-dimensional manifolds into $(n-1)$-dimensional manifolds for $n \leq 5$, whereas our proof here needs only the classification of such singular fibers for $n \leq 4$.

The paper is organized as follows. In Section 2, we review some prerequisite notions about cobordisms of manifolds and singular fibers of differentiable maps. In Section 3 , we show that $\mathfrak{N}_{2} \cong \mathbb{Z}_{2}$ and $\Omega_{2}=0$ using the classification of singular fibers of Morse functions on surfaces. We will see that the real projective plane $\mathbb{R} P^{2}$ is a natural representative of the generator of $\mathfrak{N}_{2}$ and that the Euler characteristic modulo two gives an isomorphism $\mathfrak{N}_{2} \rightarrow \mathbb{Z}_{2}$. Although the argument is quite elementary, the proof will turn out to be a good guideline for the 4-dimensional case. In Section 4 , we will show that $\Omega_{3}=0$ using the classification of singular fibers of $C^{\infty}$ stable maps of 3-manifolds into surfaces. A similar idea has been used by Costantino and D. Thurston [2008] in a proof that every 3-manifold efficiently bounds a 4-manifold. In fact, the idea of this paper is based on their work. In Section 5 , we will show that $\Omega_{4} \cong \mathbb{Z}$ by using the classification of singular fibers of $C^{\infty}$ stable maps of 4-manifolds into 3-manifolds. As a corollary, we will also show that if $f: M \rightarrow N$ is a generic differentiable map between manifolds with $\operatorname{dim} M-\operatorname{dim} N=1$ that has only singular fibers of codimension $\leq 3$ and no singular fiber of type III $^{8}$, then $M$ is null cobordant. We note that the results in this paper depend on a bundle structure theorem for singular fibers of stable maps due to Kalmár [2008, Section 5; 2009, Section 6].

Throughout the paper, all manifolds and maps are differentiable of class $C^{\infty}$. The symbol $\cong$ denotes a diffeomorphism between manifolds or an appropriate 
isomorphism between algebraic objects. For a closed surface $\Sigma$ and a positive integer $m$, we denote by $\Sigma_{(m)}$ the surface $\Sigma$ with $m$ open disks removed. We denote by $\operatorname{cl}(A)$ the closure of a subset $A$ of a topological space.

\section{Preliminaries}

Let $n$ be a nonnegative integer. Two closed oriented (possibly disconnected) $n$ dimensional manifolds $M_{0}$ and $M_{1}$ are oriented cobordant if there is a compact oriented $(n+1)$-dimensional manifold $V$ such that $\partial V=\left(-M_{0}\right) \cup M_{1}$ as oriented manifolds, where $-M_{0}$ denotes the manifold obtained by reversing the orientation of $M_{0}$. This defines an equivalence relation on the set of all closed oriented manifolds of dimension $n$, and the oriented cobordism class of a closed oriented manifold $M$ is denoted by $[M]$.

We denote by $\Omega_{n}$ the set of all oriented cobordism classes of closed oriented $n$ dimensional manifolds. This clearly forms an additive group under the operation given by $[M]+\left[M^{\prime}\right]=\left[M \cup M^{\prime}\right]$. The abelian group $\Omega_{n}$ is called the $n$-dimensional oriented cobordism group.

If we ignore the orientations of the manifolds in these definitions above, then we get the usual notion of a cobordism, and the set of all cobordism classes of closed (possibly nonorientable) $n$-dimensional manifolds is denoted by $\mathfrak{N}_{n}$, which is called the (unoriented) $n$-dimensional cobordism group.

These groups were formulated and studied in the middle of 20th century, and their structures have been completely determined. For example, see [Milnor and Stasheff 1974; Pontryagin 1955; Thom 1954; Wall 1959]. In particular, $\Omega_{n}$ and $\mathfrak{N}_{n}$ are a finitely generated $\mathbb{Z}$-module and $\mathbb{Z}_{2}$-module, respectively. (Historically, Pontrjagin [1955] first introduced such groups to compute certain homotopy groups of spheres. Thom [1954] reduced the computation of the cobordism groups to the study of homotopy groups of certain spaces, and then the structures of the cobordism groups have been determined by several authors.)

We now recall some definitions about singular fibers. See [Saeki 2004].

Definition 2.1. Let $f_{i}: M_{i} \rightarrow N_{i}$ be maps between manifolds and take points $y_{i} \in N_{i}$ for $i=0,1$. We say that the fibers over $y_{0}$ and $y_{1}$ are $C^{\infty}$ equivalent if for some open neighborhoods $U_{i}$ of $y_{i}$ in $N_{i}$, there exist diffeomorphisms $\tilde{\varphi}$ : $\left(f_{0}\right)^{-1}\left(U_{0}\right) \rightarrow\left(f_{1}\right)^{-1}\left(U_{1}\right)$ and $\varphi: U_{0} \rightarrow U_{1}$ with $\varphi\left(y_{0}\right)=y_{1}$ such that the following diagram is commutative:

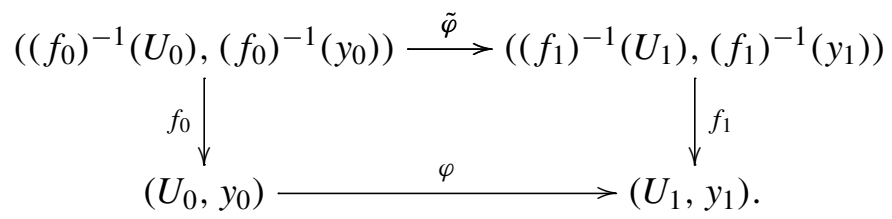


When $y \in N$ is a regular value of a map $f: M \rightarrow N$ between manifolds, we call the $C^{\infty}$ equivalence class of the fiber over $y$ (or the space $f^{-1}(y)$ ) a regular fiber; otherwise, we call it singular.

Given $f: M \rightarrow N$ and a point $y \in N$, consider the map $f \times \operatorname{id}_{\mathbb{R}}: M \times \mathbb{R} \rightarrow N \times \mathbb{R}$, where $\operatorname{id}_{\mathbb{R}}$ is the identity map of the real line $\mathbb{R}$. Then the fiber of $f \times \operatorname{id}_{\mathbb{R}}$ over the point $(y, 0) \in N \times \mathbb{R}$ is called the suspension of the fiber of $f$ over $y$.

For certain dimension pairs $(\operatorname{dim} M, \operatorname{dim} N)$, singular fibers of $C^{\infty}$ stable maps (defined below) of $M$ into $N$ have been classified up to $C^{\infty}$ equivalence. For details, see [Saeki 2004; Yamamoto 2006; Yamamoto 2007].

Definition 2.2. For manifolds $M$ and $N$, we denote by $C^{\infty}(M, N)$ the space of all smooth maps of $M$ into $N$, endowed with the Whitney $C^{\infty}$ topology. We say that a smooth map $f: M \rightarrow N$ is a $C^{\infty}$ stable map if there exists a neighborhood $U_{f}$ of $f$ in $C^{\infty}(M, N)$ such that for each $g \in U_{f}$, the diagram

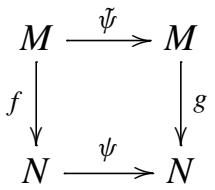

commutes for some diffeomorphisms $\psi$ and $\psi$; for details, see [Golubitsky and Guillemin 1973].

It is known that a smooth function $M \rightarrow \mathbb{R}$ on a closed manifold $M$ is $C^{\infty}$ stable if and only if it is a Morse function, that is, if and only if its critical points are all nondegenerate and have distinct critical values. Furthermore, if $\operatorname{dim} N \leq 5$, then the set of $C^{\infty}$ stable maps is open and dense in $C^{\infty}(M, N)$; see [Mather 1971].

Let us recall the following notion of a Stein factorization, which will play an important role in this paper.

Definition 2.3. Let $f: M \rightarrow N$ be a smooth map between smooth manifolds. For two points $x, x^{\prime} \in M$, we define $x \sim_{f} x^{\prime}$ if $f(x)=f\left(x^{\prime}\right)$, and the points $x$ and $x^{\prime}$ belong to the same connected component of a fiber of $f$. We define $W_{f}=M / \sim_{f}$ to be the quotient space with respect to this equivalence relation, and $q_{f}: M \rightarrow W_{f}$ denotes the quotient map. Then it is easy to see that there exists a unique continuous map $\bar{f}: W_{f} \rightarrow N$ such that the following diagram commutes:

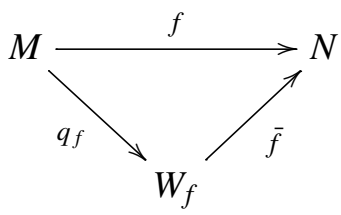

This diagram is called the Stein factorization of $f$; see [Levine 1985]. 
(1)
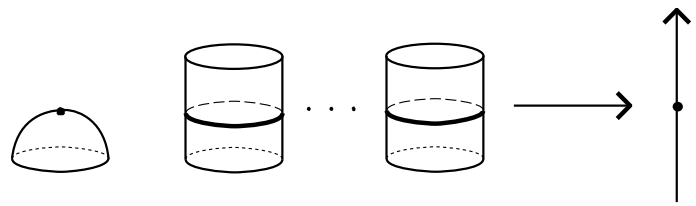

(2)
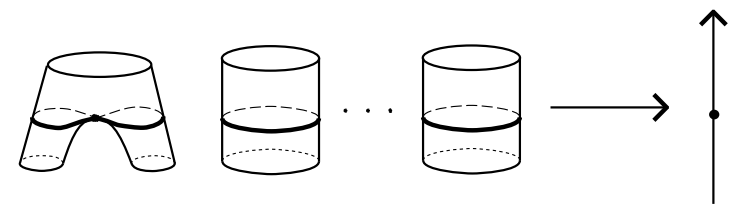

(3)
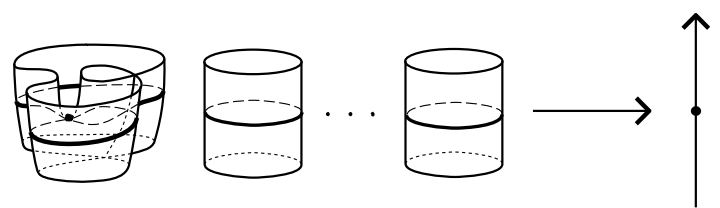

Figure 1. List of $C^{\infty}$ equivalence classes of singular fibers for Morse functions on surfaces.

If $f$ is a proper $C^{\infty}$ stable map, then $W_{f}$ is a polyhedron and all the maps appearing in this diagram are triangulable; for details, see [Hiratuka 2001].

The Stein factorization is a very useful tool for studying topological properties of $C^{\infty}$ stable maps.

\section{Two-dimensional cobordism group}

In this section, we show that $\mathfrak{N}_{2} \cong \mathbb{Z}_{2}$ and $\Omega_{2}=0$ using the classification of singular fibers of Morse functions on surfaces.

Let $M$ be an arbitrary closed 2-dimensional manifold, possibly disconnected or nonorientable. It is known that there always exists a Morse function $f: M \rightarrow \mathbb{R}$. The singular fibers of such Morse functions are classified as in Figure 1, which may be folklore; for details, see [Saeki 2004].

Construct a compact 3-dimensional manifold $V$ whose boundary includes $M$ by attaching certain pieces to $M \times[0,1]$, as follows.

Let $W_{f}$ be the quotient space in the Stein factorization of $f=\bar{f} \circ q_{f}$. It is a graph whose vertices correspond to connected components of singular fibers: the degree of a vertex is equal to 1, 3 or 2 if it corresponds to the connected component of the singular fiber as (1), (2) or (3) of Figure 1, respectively, containing the critical point; see Figure 2 for an example.

For an edge $e$ of $W_{f}$, set $e^{\prime}=\operatorname{cl}\left(e \backslash N_{0}\right)$, where $N_{0}$ is a small regular neighborhood of the set of vertices in $W_{f}$ and cl denotes the closure in $W_{f}$. Since the map 


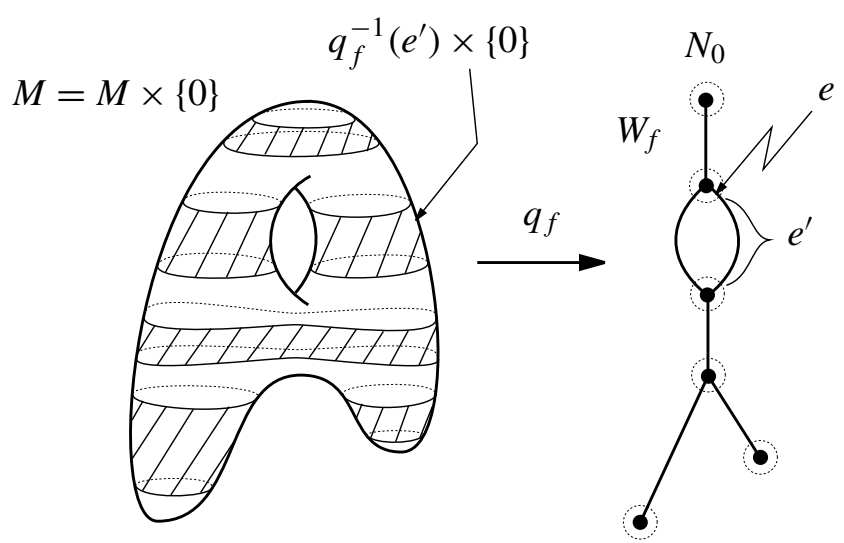

Figure 2. Constructing the 3-manifold $V$.

$q_{f}$ restricted to $q_{f}^{-1}\left(e^{\prime}\right)$ is a locally trivial fiber bundle over $e^{\prime}$ with fiber $S^{1}$, it is diffeomorphic to $S^{1} \times e^{\prime}$. Let us glue a 2-handle $D^{2} \times e^{\prime}$ to $M \times[0,1]$ by identifying $\partial D^{2} \times e^{\prime}$ and $q_{f}^{-1}\left(e^{\prime}\right) \times\{0\}$ by using the diffeomorphism above. Let us perform this operation for each edge of $W_{f}$ and denote by $V$ the resulting compact 3-dimensional manifold. For an example of the union of $M \times\{0\}$ and the 2-handles, see Figure 2.

We see that the boundary $\partial V$ is a disjoint union of $M \times\{1\}$ and some closed surfaces $F_{j}$, where each $F_{j}$ corresponds to a singular fiber of $f$. More precisely, let $v$ be a vertex of $W_{f}$ and $N_{0}(v)$ its small regular neighborhood in $W_{f}$, which is a component of $N_{0}$. Then, the corresponding surface $F_{j}$ is diffeomorphic to the union of $q_{f}^{-1}\left(N_{0}(v)\right)$ and some 2-disks attached to the regular fibers corresponding to $N_{0}(v) \cap \operatorname{cl}\left(W_{f} \backslash N_{0}\right)$.

Thus, according to the classification of singular fibers as in Figure 1, we see that each surface $F_{j}$ is connected and is diffeomorphic to $S^{2}$ for the singular fibers as in (1) and (2) of Figure 1, and to $\mathbb{R} P^{2}$ for that in Figure 1(3). See Figure 3.

Since $S^{2}=\partial D^{3}$ is null cobordant, we have proved the following.

Lemma 3.1. Every closed surface is cobordant to the disjoint union of a finite number of copies of $\mathbb{R} P^{2}$.

The cobordism class $\left[\mathbb{R} P^{2} \cup \mathbb{R} P^{2}\right]$ is zero since $\mathbb{R} P^{2} \cup \mathbb{R} P^{2}$ is the boundary of the compact 3-manifold $\mathbb{R} P^{2} \times[0,1]$. Let us consider the homomorphism

$$
\varphi: \mathbb{Z}_{2} \rightarrow \mathfrak{N}_{2}, \quad 1 \mapsto\left[\mathbb{R} P^{2}\right],
$$

where $1 \in \mathbb{Z}_{2}$ is the generator. This is a well-defined homomorphism, and is surjective by Lemma 3.1. 
(1)

Singular fiber

$F_{j}$
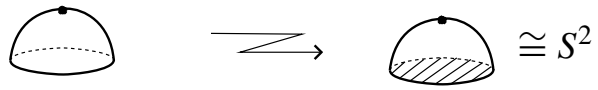

(2)
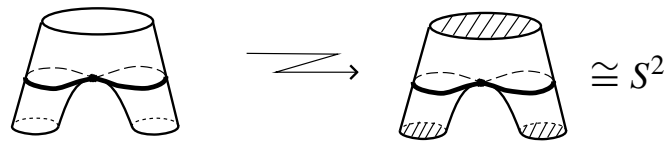

(3)
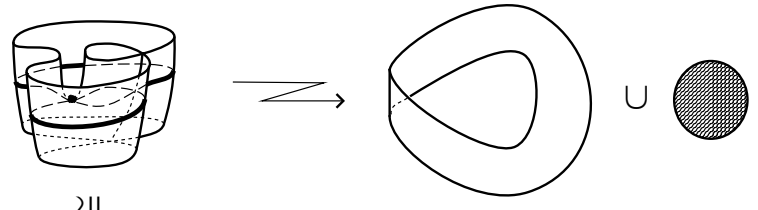

2II

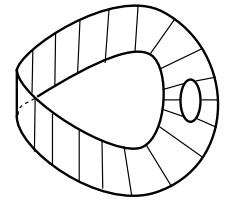

2II

$\mathbb{R} P^{2}$

Figure 3. Surface $F_{j}$ appearing around each singular fiber.

Let

$$
\chi_{2}: \mathfrak{N}_{2} \rightarrow \mathbb{Z}_{2}
$$

be the homomorphism defined by associating to each cobordism class the Euler characteristic modulo two of its representative. Using standard techniques in algebraic topology, we can show that this defines a well-defined homomorphism; for example, see [Thom 1952].

Since the Euler characteristic of $\mathbb{R} P^{2}$ is equal to 1 , we see that the composition $\chi_{2} \circ \varphi: \mathbb{Z}_{2} \rightarrow \mathbb{Z}_{2}$ is the identity. Therefore, $\varphi$ must be injective. Thus, we have proved the following.

Theorem 3.2. The 2-dimensional cobordism group $\mathfrak{N}_{2}$ is cyclic of order two and is generated by the cobordism class of $\mathbb{R} P^{2}$. In fact, the homomorphism (3-1) is an isomorphism.

Our proof does not depend on the classification of closed surfaces.

As a corollary to the proof, we also get the following, which was originally obtained in [Saeki 2004]. 


$$
\begin{aligned}
& \kappa=1 \quad \mathrm{I}^{0} \bullet \quad \mathrm{I}^{1} \infty \\
& \kappa=2 \quad \mathrm{II}^{2} 8 \quad \mathrm{II}^{3} \bigcirc \quad \mathrm{II}^{a} \bigcirc
\end{aligned}
$$

Figure 4. List of $C^{\infty}$ equivalence classes of singular fibers of $C^{\infty}$ stable maps of orientable 3-manifolds into surfaces.

Corollary 3.3. Let $f: M \rightarrow \mathbb{R}$ be a Morse function on a closed surface $M$. Then, the number of singular fibers of $f$ as in Figure 1(3) has the same parity as the Euler characteristic of $M$.

If a closed surface $M$ is oriented, then a singular fiber as in Figure 1(3) never appears, since its neighborhood is nonorientable. Furthermore, the 3-manifold $V$ constructed above is orientable, since $M \times[0,1]$ is orientable and attaching a 2handle does not alter the orientability of a 3-manifold. Moreover, $V$ can be oriented so that its oriented boundary consists of $M$ and some 2-spheres. Thus:

Corollary 3.4. The 2-dimensional oriented cobordism group $\Omega_{2}$ vanishes.

\section{Three-dimensional oriented cobordism group}

In this section, we show that $\Omega_{3}=0$ by using the classification of singular fibers of $C^{\infty}$ stable maps of closed orientable 3-manifolds into surfaces.

For $M$ a closed oriented 3-manifold, there always exists a $C^{\infty}$ stable map $f$ from $M$ into any surface $N$; for example, see [Kushner et al. 1984; Levine 1985]. Singular fibers of such maps have been classified in [Saeki 2004] up to $C^{\infty}$ equivalence; see also [Kushner et al. 1984; Levine 1985]. The connected components of singular fibers containing singular points are as depicted in Figure 4, where each figure represents the connected component of the inverse image of a point in the target. (In fact, it is known that two such singular fibers are $C^{\infty}$ equivalent if and only if the corresponding inverse images are diffeomorphic to each other. For details, see [Saeki 2004].) Furthermore, in Figure 4, $\kappa$ denotes the codimension of a singular fiber, that is, it is the codimension of the set of those points in the target over which lies a relevant singular fiber. Following the convention introduced in [Saeki 2004], we use the notation $\mathrm{I}^{0}, \mathrm{I}^{1}$ and so on for the $C^{\infty}$ equivalence classes of singular fibers. The $\mathrm{I}^{0}$-type (or $\mathrm{I}^{1}$-type) singular fiber is the suspension of the singular fiber for Morse functions as in Figure 1(1) (respectively Figure 1(2)).

A $C^{\infty}$ stable map of a 3-manifold into a surface has only fold and cusp singularities [Kushner et al. 1984; Levine 1985]. A singular fiber (or the corresponding inverse image) can be regarded as a graph: a fold point corresponds to an isolated 
vertex or to a vertex of degree four, and a cusp point corresponds to a cuspidal vertex of degree two (see Figure 4).

Let $W_{f}$ be the quotient space in the Stein factorization of a $C^{\infty}$ stable map $f$ from a closed oriented 3-manifold $M$ into a surface $N$. The space $W_{f}$ is a compact 2-dimensional polyhedron and its local structure is completely determined [Kushner et al. 1984; Levine 1985]. Let $W^{(0)}$ denote the $q_{f}$-image of the singular fibers of $\kappa=2$, and let $W^{(1)}$ denote the $q_{f}$-image of the singular fibers of $\kappa \geq 1$ (more precisely, they are the $q_{f}$-images of the components of the relevant singular fibers containing singular points). Note that $W^{(0)}$ is a finite set of points and $W^{(1)}$ is a 1-dimensional subcomplex of $W_{f}$ whose complement is a nonsingular surface. For $i=0,1$, we denote by $N^{(i)}$ a small regular neighborhood of $W^{(i)}$ in $W_{f}$. We set $N_{0}=N^{(0)}, N_{1}=\operatorname{cl}\left(N^{(1)} \backslash N^{(0)}\right)$ and $N_{2}=\operatorname{cl}\left(W_{f} \backslash N^{(1)}\right)$, where $N_{1}$ is regarded as a regular neighborhood of $\operatorname{cl}\left(W^{(1)} \backslash N^{(0)}\right)$ in $\operatorname{cl}\left(W_{f} \backslash N^{(0)}\right)$. Note that $W_{f}$ is decomposed as

$$
W_{f}=N_{0} \cup N_{1} \cup N_{2} .
$$

Let us construct a compact 4-dimensional manifold $V$ by attaching certain pieces to $M \times[0,1]$ as follows. First note that $q_{f}$ restricted to $q_{f}^{-1}\left(N_{2}\right)$ is a locally trivial fibration with fiber $S^{1}$ over the surface $N_{2}$. Thus, we can attach the total space of the associated $D^{2}$-bundle over $N_{2}$ to $M \times[0,1]$ by identifying the associated $\left(\partial D^{2}\right.$ )bundle with $q_{f}^{-1}\left(N_{2}\right) \times\{0\}$. (Here, we use the well-known fact that the structure group of every smooth $S^{1}$-bundle can be reduced to the orthogonal group $O(2)$.) The resulting 4-manifold is denoted by $V_{1}$. Note that $V_{1}$ is orientable, since $M$ and $q_{f}^{-1}\left(N_{2}\right)$ are orientable as 3-manifolds.

Let $V_{1}^{\prime}\left(\subset V_{1}\right)$ denote the union of $M \times\{0\}$ and the $D^{2}$-bundle over $N_{2}$. There is a natural map $q_{1}: V_{1}^{\prime} \rightarrow W_{f}$, which on $M \times\{0\}$ is defined by $q_{f}: M \times\{0\}=M \rightarrow W_{f}$, and on the $D^{2}$-bundle is defined by the projection to $N_{2}$.

Let $e$ be a connected component of $\operatorname{cl}\left(W^{(1)} \backslash N_{0}\right)$. Note that $e$ is an arc or a circle. Let $N_{1}(e)$ denote the connected component of $N_{1}$ containing $e$. If the singular fiber lying over a point in $e$ is of type $\mathrm{I}^{0}$, then $q_{f}^{-1}\left(N_{1}(e)\right)$ is diffeomorphic to the total space of a $D^{2}$-bundle over $e$ [Kushner et al. 1984; Levine 1985]. In fact, $N_{1}(e)$ is diffeomorphic to $e \times[0,1]$ by a diffeomorphism that induces the identity $e\left(\subset N_{1}(e)\right) \rightarrow e \times\{0\}$, and if $J(\cong[0,1])$ is a fiber of the natural fibration $N_{1}(e) \rightarrow e$, then $q_{f}$ restricted to $q_{f}^{-1}(J) \cong D^{2}$ is equivalent to the function

$$
(x, y) \mapsto x^{2}+y^{2} .
$$

(In fact, the commutative diagram

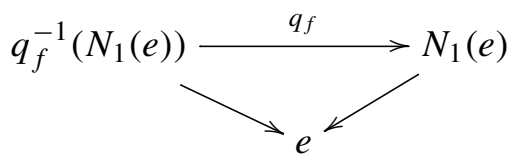


can be regarded as a fiber bundle with the map $D^{2} \rightarrow[0,1]$ defined by (4-1) as fiber in an appropriate sense. For details, see [Kalmár 2008, Section 5; Kalmár 2009, Section 6].)

Therefore, $q_{1}$ restricted to $q_{1}^{-1}\left(N_{1}(e)\right)$ followed by the natural projection from $N_{1}(e)$ to $e$ is an $S^{2}$-bundle, where the fiber of this fibration can be identified with the 2-sphere as in Figure 3(1) in Section 3. Then, we can attach the associated $D^{3}$-bundle over $e$ to $V_{1}$, where we identify the associated $\left(\partial D^{3}\right)$-bundle with $q_{1}^{-1}\left(N_{1}(e)\right)$. Here, we use the fact that the structure group of every smooth $S^{2}$-bundle can be reduced to $O(3)$; see [Smale 1959]. Note that the resulting 4manifold is orientable, since so is $V_{1}$ and the orientability of $q_{1}^{-1}\left(N_{1}(e)\right)$ coincides with that of $q_{f}^{-1}\left(N_{1}(e)\right)(\subset M)$.

If the singular fiber lying over a point in $e$ is of type $\mathrm{I}^{1}$, then the natural projection $N_{1}(e) \rightarrow e$ defines a $Y$-bundle, where

$$
Y=\{r \exp (2 \pi \sqrt{-1} k / 3) \in \mathbb{C} \mid 0 \leq r \leq 1, k=0,1,2\} .
$$

Moreover, $q_{f}^{-1}\left(N_{1}(e)\right)$ is diffeomorphic to the total space of an $S_{(3)}^{2}$-bundle over $e$, where $S_{(3)}^{2}$ denotes the 2-sphere with three open disks removed. Then, $q_{1}$ restricted to $q_{1}^{-1}\left(N_{1}(e)\right)$ followed by the natural projection $N_{1}(e) \rightarrow e$ is again an $S^{2}$-bundle, but with fiber as in Figure 3(2), and we can attach the associated $D^{3}$-bundle.

We perform the operation described above for each $e$. The resulting 4-manifold, denoted by $V$, is a compact 4-dimensional manifold that is orientable. Furthermore, it can be oriented so that

$$
\partial V=(M \times\{1\}) \cup\left(-\bigcup_{j} F_{j}\right),
$$

where each $F_{j}$ is a closed oriented 3-manifold corresponding to a singular fiber of $f$ of $\kappa=2$.

Lemma 4.1. The closed 3-manifold $F_{j}$ is diffeomorphic to the 3-sphere $S^{3}$ for every singular fiber of $\kappa=2$.

In fact, for the singular fibers of types $\mathrm{II}^{2}$ and $\mathrm{II}^{3}$, this lemma has been essentially obtained in [Costantino and Thurston 2008]. Here we give a proof from a different viewpoint in a way that is useful in Section 5.

Proof. Let $v$ be a point in $W_{f}$ that is the $q_{f}$-image of a singular fiber of type $\mathrm{II}^{2}$. Then, its regular neighborhood $N_{0}(v)$ in $W_{f}$ is of the form depicted in Figure 5, where $N_{0}(v)$ is the component of $N_{0}$ containing $v$. Note that $\bar{f}\left(N_{0}(v)\right) \cong J_{1} \times J_{2}$ with $J_{1}=J_{2}=[-1,1]$.

Then, the map $f$ restricted to $q_{f}^{-1}\left(N_{0}(v)\right)$ can be regarded as a 1-parameter family of functions on $S_{(4)}^{2}$ with only nondegenerate critical points as depicted in Figure 6 . This family is parametrized by $J_{1}$, where for each parameter value the relevant function is regarded as a height function $S_{(4)}^{2} \rightarrow J_{2}$. 


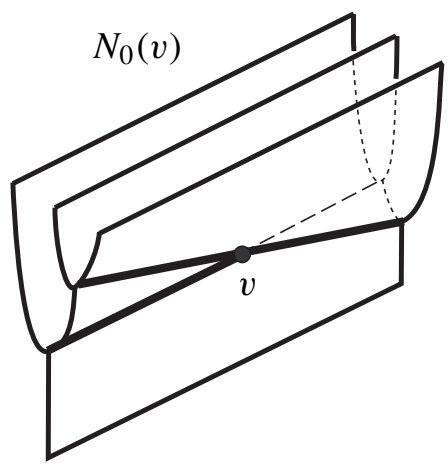

Figure 5. Neighborhood of the $q_{f}$-image of a $\mathrm{II}^{2}$-type singular fiber.

In constructing $V_{1}$, we attached to each $S_{(4)}^{2}$ 2-disks along the four boundary circles so that we get a 2-sphere. Along the 2 -spheres for $t= \pm 1$, we attached 3-disks to construct $V$. Thus, the relevant 3-manifold $F_{j}$ is diffeomorphic to a manifold obtained by attaching two 3 -disks to $S^{2} \times[-1,1]$ along the boundaries, and is therefore diffeomorphic to the 3 -sphere $S^{3}$.

The same argument can be applied for the singular fiber of type $\mathrm{II}^{3}$. The regular neighborhood of a corresponding point in $W_{f}$ is shown in Figure 7.

Finally, for the singular fiber of type $\mathrm{II}^{a}$, a similar argument can be applied as follows. The regular neighborhood of a corresponding point in $W_{f}$ is shown in the top of Figure 8 . The map $f$ restricted to the inverse image of the neighborhood

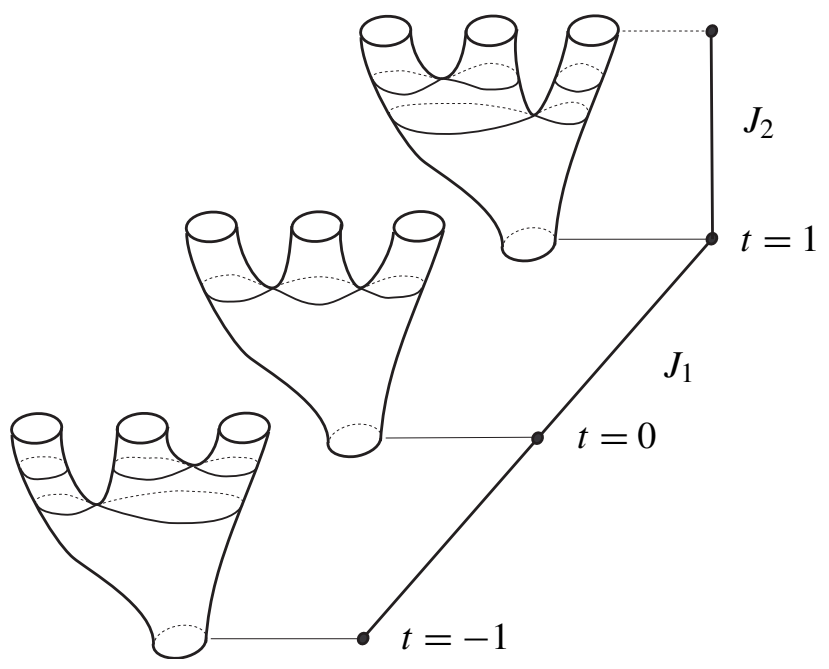

Figure 6. 1-parameter family of functions on $S_{(4)}^{2}$ corresponding to a $\mathrm{II}^{2}$-type singular fiber. 


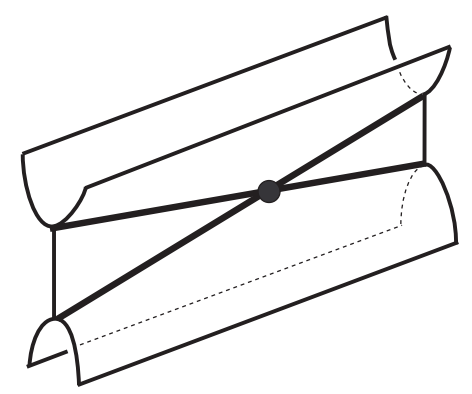

Figure 7. Neighborhood of the $q_{f}$-image of a $\mathrm{II}^{3}$-type singular fiber.

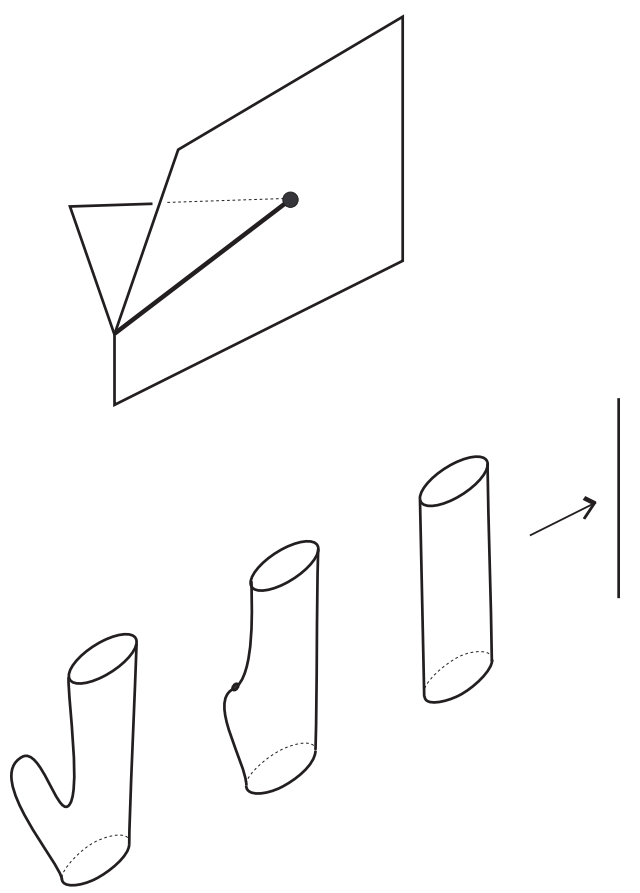

Figure 8. The case of a singular fiber of type $\mathrm{II}^{a}$.

with respect to $q_{f}$ can be regarded as a 1-parameter family of smooth functions on the annulus corresponding to a birth-death of a pair of nondegenerate critical points as shown in the bottom of Figure 8 . Thus, the resulting 3 -manifold $F_{j}$ is again diffeomorphic to $S^{3}$.

This completes the proof of Lemma 4.1.

Since $S^{3}$ is the oriented boundary of an oriented 4-disk, we see that $M$ bounds a compact oriented 4-manifold. Therefore, we have proved the following. 


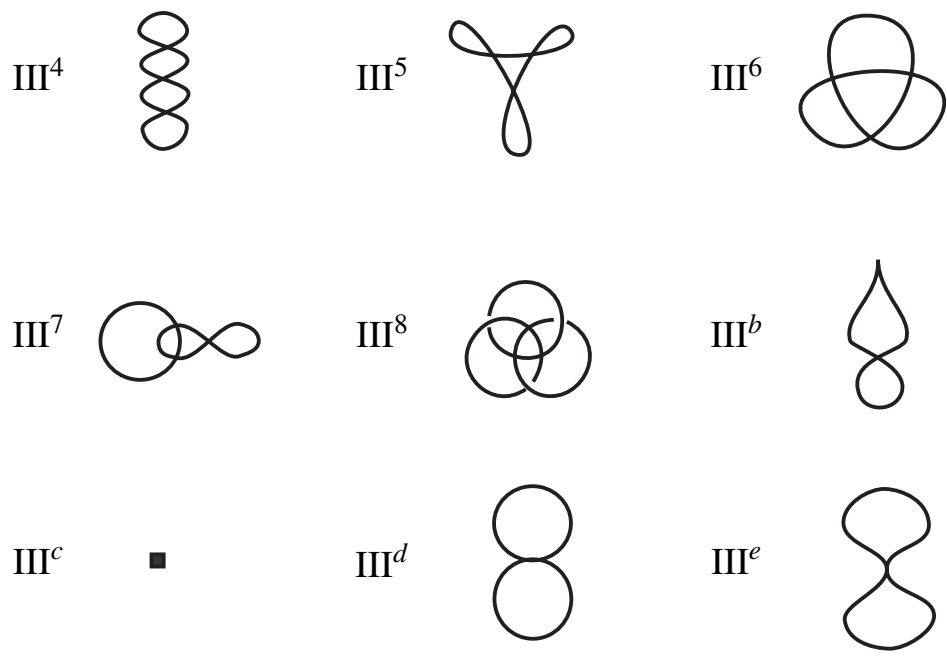

Figure 9. Singular fibers of $C^{\infty}$ stable maps of orientable 4manifolds into 3-manifolds of $\kappa=3$.

Theorem 4.2. The 3-dimensional oriented cobordism group $\Omega_{3}$ vanishes.

This result is originally due to Rohlin [1951] and Thom [1952]. In fact, our argument resembles that in [Costantino and Thurston 2008].

Remark 4.3. Kalmár [2009] showed that for every $C^{\infty}$ stable map $f$ of a closed orientable 3-manifold $M$ into the plane, singular fibers of types $\mathrm{II}^{2}, \mathrm{II}^{3}$ and $\mathrm{II}^{a}$ can be eliminated by cobordism. Using this result, he showed that $\Omega_{3}=0$. For details, see [Kalmár 2009, Remark 2.8].

\section{Four-dimensional oriented cobordism group}

In this section, we show that the oriented cobordism group $\Omega_{4}$ is infinite cyclic and is generated by the cobordism class of $\mathbb{C} P^{2}$, using the classification of singular fibers of $C^{\infty}$ stable maps of orientable 4-manifolds into 3-manifolds.

For $M$ a closed oriented 4-manifold, there always exists a $C^{\infty}$ stable map $f$ from $M$ into any 3-manifold $N$; for example, see [Saeki 2004]. Singular fibers of such maps were classified up to $C^{\infty}$ equivalence in [Saeki 2004]. The connected components of singular fibers containing singular points are shown in Figure 9 in addition to the singular fibers of codimension $\kappa=1$ and 2 that are suspensions of the singular fibers as in Figure 4. (For the singular fibers of $\kappa \leq 2$, we continue to use the same notation $\mathrm{I}^{0}, \mathrm{I}^{1}$, and so on as in Figure 4 for the suspensions as well.)

A $C^{\infty}$ stable map of a 4-manifold into a 3-manifold has only fold, cusp, and swallowtail singularities. A fold point corresponds to an isolated point or to a transverse crossing point of two line segments, a cusp point corresponds to a cuspidal 
vertex of degree two in a singular fiber, and a swallowtail point corresponds to an isolated point (depicted by a black square in Figure 9, $\mathrm{III}^{c}$ ) or to a tangency point of two touching parabolas.

Let $W_{f}$ be the quotient space in the Stein factorization of a $C^{\infty}$ stable map $f$ of a closed oriented 4-manifold $M$ into a 3-manifold $N$. The space $W_{f}$ is a compact 3-dimensional polyhedron, and its local structure has been completely determined. A complete list of local structures can be found in [Hiratuka 2001], although we do not need it here. Let $W^{(j)}$ denote the $q_{f}$-image of the components of singular fibers of $\kappa \geq 3-j$ containing singular points for $j=0,1,2$, and let $N^{(j)}$ denote a small regular neighborhood of $W^{(j)}$ in $W_{f}$. Then, set

$$
N_{0}=N^{(0)}, \quad N_{1}=\operatorname{cl}\left(N^{(1)} \backslash N^{(0)}\right), \quad N_{2}=\operatorname{cl}\left(N^{(2)} \backslash N^{(1)}\right), \quad N_{3}=\operatorname{cl}\left(W_{f} \backslash N^{(2)}\right),
$$

where $N_{1}$ is seen as a regular neighborhood of $\operatorname{cl}\left(W^{(1)} \backslash N^{(0)}\right)$ in $\operatorname{cl}\left(W_{f} \backslash N^{(0)}\right)$ and $N_{2}$ is seen as a regular neighborhood of $\operatorname{cl}\left(W^{(2)} \backslash N^{(1)}\right)$ in $\operatorname{cl}\left(W_{f} \backslash N^{(1)}\right)$.

Let us now construct a compact 5-dimensional manifold $V$ by attaching certain pieces to $M \times[0,1]$ as follows. First note that $q_{f}$ restricted to $q_{f}^{-1}\left(N_{3}\right)$ is a locally trivial fibration with fiber $S^{1}$ over the 3-manifold possibly with boundary $N_{3}$. Thus, we can attach the total space of the associated $D^{2}$-bundle over $N_{3}$ to $M \times[0,1]$ by identifying the associated $\left(\partial D^{2}\right)$-bundle with $q_{f}^{-1}\left(N_{3}\right) \times\{0\}$. The resulting 5-manifold, denoted by $V_{1}$, is orientable.

Let $V_{1}^{\prime}$ (a subset of $V_{1}$ ) be the union of $M \times\{0\}$ and the $D^{2}$-bundle over $N_{3}$. There is a natural map $q_{1}: V_{1}^{\prime} \rightarrow W_{f}$ that on $M \times\{0\}$ is defined by $q_{f}: M \times\{0\}=M \rightarrow W_{f}$, and on the $D^{2}$-bundle is defined by the projection to $N_{3}$.

Let $S$ be a connected component of $\operatorname{cl}\left(W^{(2)} \backslash N^{(1)}\right)$. Note that $S$ is a compact surface possibly with boundary. Let $N_{2}(S)$ denote the connected component of $N_{2}$ containing $S$. If the singular fiber lying over a point in $S$ is of type $\mathrm{I}^{0}$, then $q_{f}^{-1}\left(N_{2}(S)\right)$ is diffeomorphic to the total space of a $D^{2}$-bundle over $S$. In fact, $N_{2}(S)$ is diffeomorphic to $S \times[0,1]$ by a diffeomorphism that induces the identity $S\left(\subset N_{2}(S)\right) \rightarrow S \times\{0\}$, and if $J(\cong[0,1])$ is a fiber of the natural fibration $N_{2}(S) \rightarrow S$, then $q_{f}$ restricted to $q_{f}^{-1}(J) \cong D^{2}$ is equivalent to the function (4-1). (For this, we need the bundle structure theorem mentioned in [Kalmár 2008, Section 5; Kalmár 2009, Section 6].) Therefore, the map $q_{1}$ restricted to $q_{1}^{-1}\left(N_{2}(S)\right)$ followed by the natural projection $N_{2}(S) \rightarrow S$ is an $S^{2}$-bundle whose fiber can be identified with the 2-sphere as in Figure 3(1). Then, we can attach the associated $D^{3}$-bundle over $S$ to $V_{1}$, where we identify the associated $\left(\partial D^{3}\right)$-bundle with $q_{1}^{-1}\left(N_{2}(S)\right)\left(\subset V_{1}^{\prime}\right)$. The resulting 5-manifold is orientable, since so is $V_{1}$ and the orientability of $q_{1}^{-1}\left(N_{2}(S)\right)$ coincides with that of $q_{f}^{-1}\left(N_{2}(S)\right)(\subset M)$.

If the singular fiber lying over a point in $S$ is of type $\mathrm{I}^{1}$, then $q_{f}^{-1}\left(N_{2}(S)\right)$ is diffeomorphic to the total space of an $S_{(3)}^{2}$-bundle over $S$. Then, $q_{1}$ restricted to $q_{1}^{-1}\left(N_{2}(S)\right)$ followed by the natural projection $N_{2}(S) \rightarrow S$ is again an $S^{2}$-bundle, 

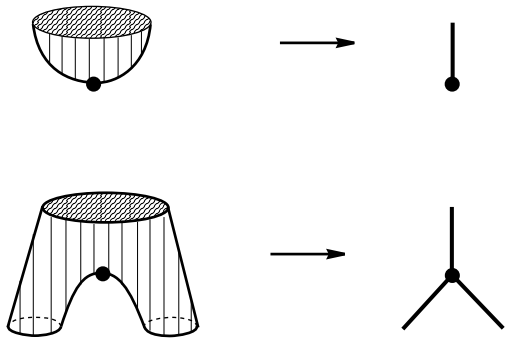

Figure 10. Function on each $D^{3}$-fiber.

but with fiber as in Figure 3(2), and we can attach the associated $D^{3}$-bundle. As before, the resulting 5-manifold is orientable.

We perform the operation described above for each connected component $S$ of $\operatorname{cl}\left(W^{(2)} \backslash N^{(1)}\right)$. The resulting 5-manifold, denoted $V_{2}$, is orientable. We denote by $V_{2}^{\prime}$ the union of $V_{1}^{\prime}$ and the $D^{3}$-bundle over $\operatorname{cl}\left(W^{(2)} \backslash N^{(1)}\right)$. There is a natural map $q_{2}: V_{2}^{\prime} \rightarrow W_{f}$ that on $V_{1}^{\prime}$ is defined by $q_{1}: V_{1}^{\prime} \rightarrow W_{f}$, and on the $D^{3}$-bundle $X$ over $\operatorname{cl}\left(W^{(2)} \backslash N^{(1)}\right)$ is defined by the natural map $X \rightarrow N_{2}$ that makes the diagram

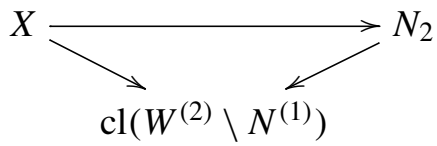

commutative and is given by the function on each fiber as shown in Figure 10. (For this, we again need Kalmár's bundle structure theorem.)

Let $e$ be a connected component of $\operatorname{cl}\left(W^{(1)} \backslash N^{(0)}\right)$ and we denote by $N_{1}(e)$ the connected component of $N_{1}$ containing $e$. If the singular fiber lying over a point in $e$ is of type $\mathrm{II}^{2}$, then $q_{f}^{-1}\left(N_{1}(e)\right)$ is diffeomorphic to the total space of an $\left(S_{(4)}^{2} \times[0,1]\right)$-bundle over $e$; see Figure 6 .

Therefore, $q_{2}$ restricted to $q_{2}^{-1}\left(N_{1}(e)\right)$ followed by the natural projection from $N_{1}(e)$ to $e$ is an $S^{3}$-bundle by Lemma 4.1. From [Hatcher 1983], the structure group of every smooth $S^{3}$-bundle can be reduced to the orthogonal group $O(4)$. Then, we can attach the associated $D^{4}$-bundle over $e$ to $V_{2}$, where we identify the associated $\left(\partial D^{4}\right)$-bundle with $q_{2}^{-1}\left(N_{1}(e)\right)\left(\subset V_{2}^{\prime}\right)$. The resulting 5-manifold is orientable, since so is $V_{2}$ and the orientability of $q_{2}^{-1}\left(N_{1}(e)\right)$ coincides with that of $q_{f}^{-1}\left(N_{1}(e)\right)(\subset M)$.

If the singular fiber lying over a point in $e$ is of another type $\left(\mathrm{II}^{3}\right.$ or $\left.\mathrm{II}^{a}\right)$, then we can still perform the same operation by virtue of Lemma 4.1.

We perform such an operation for each $e$. The resulting 5-manifold $V$ is compact and orientable. Note that $V$ can be oriented so that

$$
\partial V=(M \times\{1\}) \cup\left(-\bigcup_{j} F_{j}\right),
$$


where each $F_{j}$ is a closed oriented 4-manifold corresponding to a singular fiber of $f$ of $\kappa=3$.

Lemma 5.1. $F_{j}$ is diffeomorphic to the 4-sphere $S^{4}$ for the singular fibers of types $\mathrm{III}^{4}, \mathrm{III}^{5}, \mathrm{III}^{6}, \mathrm{III}^{7}, \mathrm{III}^{b}, \mathrm{III}^{c}, \mathrm{III}^{d}$ and $\mathrm{III}^{e}$.

Proof. Let us first consider the case of the singular fiber of type $\mathrm{III}^{4}$. Let $g: L \rightarrow D^{3}$ be a representative of the singular fiber; we assume that it has a singular fiber of type $\mathrm{III}^{4}$ over the center of $D^{3}$. Then, we can regard $g$ as a family of functions $\left\{h_{s}\right\}_{s \in \Delta}$ on $S_{(5)}^{2}$ with only nondegenerate critical points parametrized by $\Delta \cong D^{2}$ as depicted in Figure 11, where the target $D^{3}$ is identified with the product $[-1,1] \times \Delta$, $\pi:[-1,1] \times \Delta \rightarrow \Delta$ is the projection to the second factor, and the critical points of $h_{s}$ are denoted by $p_{1}, p_{2}$ and $p_{3}$. More precisely, $g$ can be identified with the map $L \cong S_{(5)}^{2} \times \Delta \rightarrow[-1,1] \times \Delta \cong D^{3},(x, s) \mapsto\left(h_{s}(x), s\right)$. The singular point set $S(g)$ of $g$ consists of three 2-disks, and their images by $g$ in $D^{3}$ intersect at the origin in general position.

Then, from the construction of $V$ it follows that the 4-manifold $F_{j}$ corresponding to $g^{-1}(0)$ is diffeomorphic to the boundary of a $\widetilde{D}$-bundle over $\Delta$, where $\widetilde{D} \cong D^{3}$ is the 3 -disk as in Figure 12, which is obtained by filling $S_{(5)}^{2} \times[0,1]$ by 2 - and 3-handles as in Section 3. Hence $F_{j}$ is diffeomorphic to $S^{4}$.

For the singular fibers of types $\mathrm{III}^{5}, \mathrm{III}^{b}, \mathrm{III}^{c}$ and $\mathrm{III}^{e}$, similar arguments show that $F_{j} \cong S^{4}$.

For the singular fiber of type $\mathrm{III}^{6}$, we can again regard its representative $g$ : $L \rightarrow D^{3}$ as a family of functions parametrized by a 2-disk $\Delta$. Note that $L$ is diffeomorphic to $S_{(5)}^{2} \times \Delta$. Set $F_{j}^{\prime}=L \cup q_{1}^{-1}\left((\bar{g})^{-1}(\{ \pm 1\} \times \Delta)\right)$ and $F_{j}^{\prime \prime}=\operatorname{cl}\left(F_{j} \backslash F_{j}^{\prime}\right)$, where $g=\bar{g} \circ q_{g}$ is the Stein factorization of $g: L \rightarrow[-1,1] \times \Delta$ and we regard $W_{g}$ as a subset of $W_{f}$.

Then, we see that $F_{j}^{\prime}$ is diffeomorphic to $S^{2} \times D^{2}$. On the other hand, from the construction of $V$ it follows that $F_{j}^{\prime \prime}$ is the union of six copies of $D^{3} \times[-1,1]$ attached to each other along $D^{3} \times\{ \pm 1\}$ consecutively so that if forms the total space of a $D^{3}$-bundle over $S^{1}$. Hence, $F_{j}^{\prime \prime}$ is diffeomorphic to $D^{3} \times S^{1}$, since it is orientable. Therefore, $F_{j}$ is diffeomorphic to the union of $S^{2} \times D^{2}$ and $D^{3} \times S^{1}$ attached along their boundaries, where $S^{2} \times\{*\} \subset S^{2} \times D^{2}$ and $\partial D^{3} \times\{*\} \subset D^{3} \times S^{1}$ are identified. Then a standard argument shows that $F_{j}$ is diffeomorphic to $S^{4}$.

For the singular fibers of types $\mathrm{III}^{7}$ and $\mathrm{III}^{d}$, similar arguments give $F_{j} \cong S^{4}$.

This completes the proof of Lemma 5.1.

Lemma 5.2. For the singular fiber of type $\mathrm{III}^{8}, F_{j}$ is orientation-preservingly diffeomorphic to the complex projective plane $\mathbb{C} P^{2}$ or its orientation reversal $\overline{\mathbb{C} P^{2}}$.

Proof. As in the proof of Lemma 5.1, a representative $g: L \rightarrow D^{3}$ of the singular fiber of type $\mathrm{III}^{8}$ can be regarded as a family of functions $\left\{h_{s}\right\}_{s \in \Delta}$ on $T_{(3)}^{2}$ with only nondegenerate critical points parametrized by a small 2-disk $\Delta$. (Here, the torus 

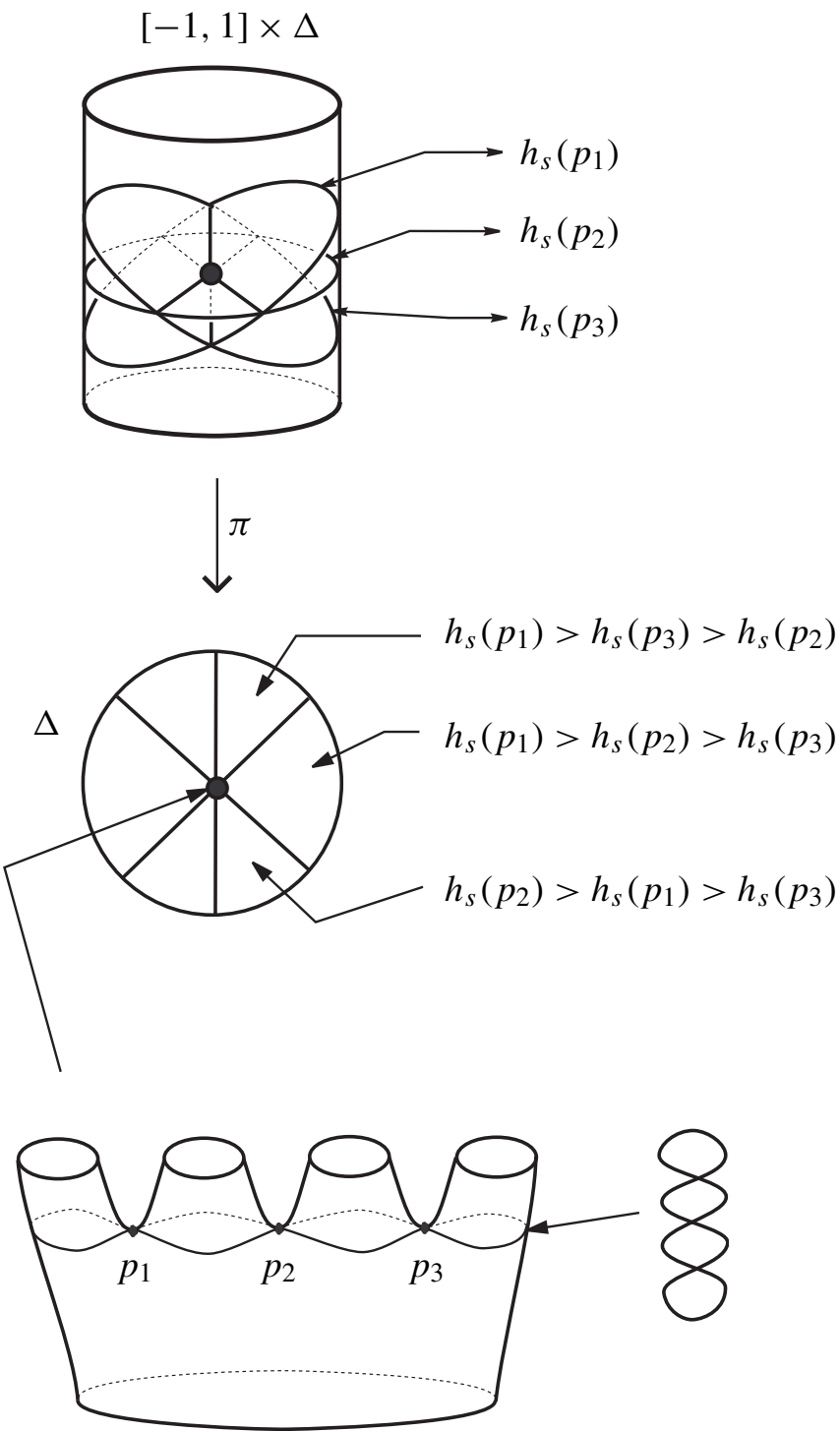

Figure 11. Family of functions corresponding to a singular fiber of type $\mathrm{III}^{4}$.

with three holes appears, since the natural "thickening" of the $\mathrm{III}^{8}$-type singular fiber is a compact orientable surface with three boundary circles and has Euler characteristic -3 . See Figure 13.) See Figure 14, where $\pi: D^{3} \cong[-1,1] \times \Delta \rightarrow \Delta$ is the projection onto the second factor; see also [Saeki 2004, Figure 6.3].

Set $K=g^{-1}\left(\partial D^{3}\right)=\partial L$, which is a closed orientable 3-manifold. Then, $\left.g\right|_{K}$ : $K \rightarrow \partial D^{3}$ can be regarded as a stable map as in Section 4. Note that $F_{j}$ is $L \cup W$ 


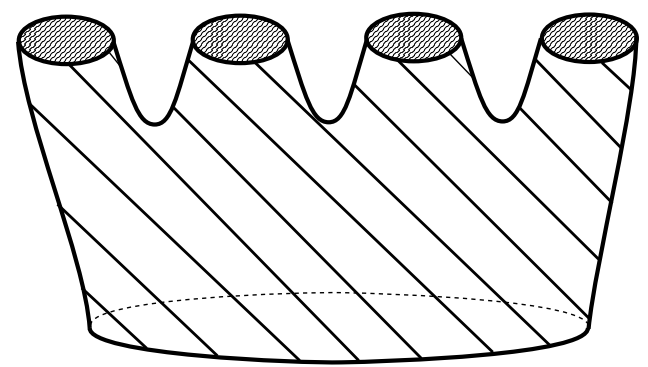

Figure 12. $\widetilde{D} \cong D^{3}$.

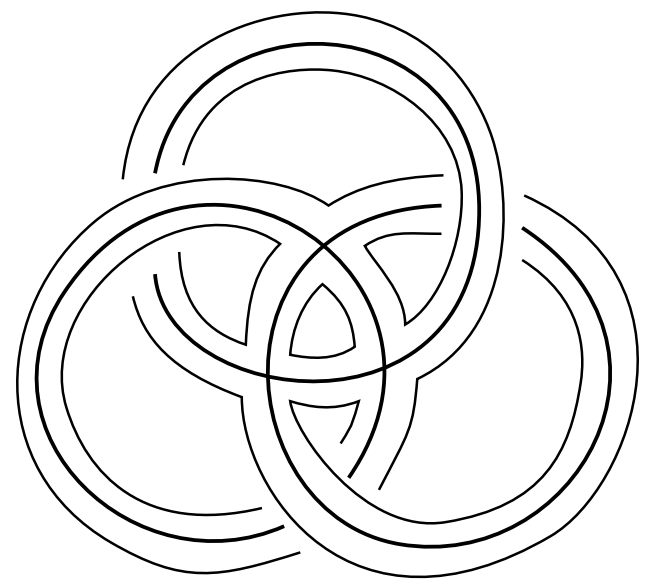

Figure 13. Natural "thickening" of the $\mathrm{III}^{8}$-type singular fiber.

attached along $K$, where $W$ is the compact orientable 4-manifold bounded by $K$ constructed as in Section 4 from the stable map $\left.g\right|_{K}$.

Set

$$
F_{j}^{\prime}=L \cup q_{1}^{-1}\left((\bar{g})^{-1}(\{ \pm 1\} \times \Delta)\right) \quad \text { and } \quad F_{j}^{\prime \prime}=\operatorname{cl}\left(F_{j} \backslash F_{j}^{\prime}\right)
$$

as in the proof of the previous lemma. Note that $F_{j}^{\prime}$ is diffeomorphic to a $T^{2}$-bundle over $\Delta$. (More precisely, the map $\pi \circ g: L \rightarrow \Delta$ is a smooth fiber bundle with $T_{(3)}^{2}$ as fibers, and $F_{j}^{\prime}$ is obtained from $L$ by attaching three 2-disks to each of the fibers.)

Let us consider the piece $P_{i}$ in $F_{j}^{\prime \prime}$ corresponding to the arc $\alpha_{i}$ for $i=1,2,3$, on $\partial \Delta$ as depicted in Figure 14. More precisely, $P_{i}$ is the compact 4-manifold described as follows. First, note that $\left.g\right|_{(\pi \circ g)^{-1}\left(\alpha_{i}\right)}:(\pi \circ g)^{-1}\left(\alpha_{i}\right) \rightarrow[-1,1] \times \alpha_{i}$ can be regarded as a 1-parameter family of smooth functions on $T_{(3)}^{2}$ with exactly three nondegenerate critical points corresponding to interchanging the heights of the top two critical points. Furthermore, the singular fiber (of codimension two) 

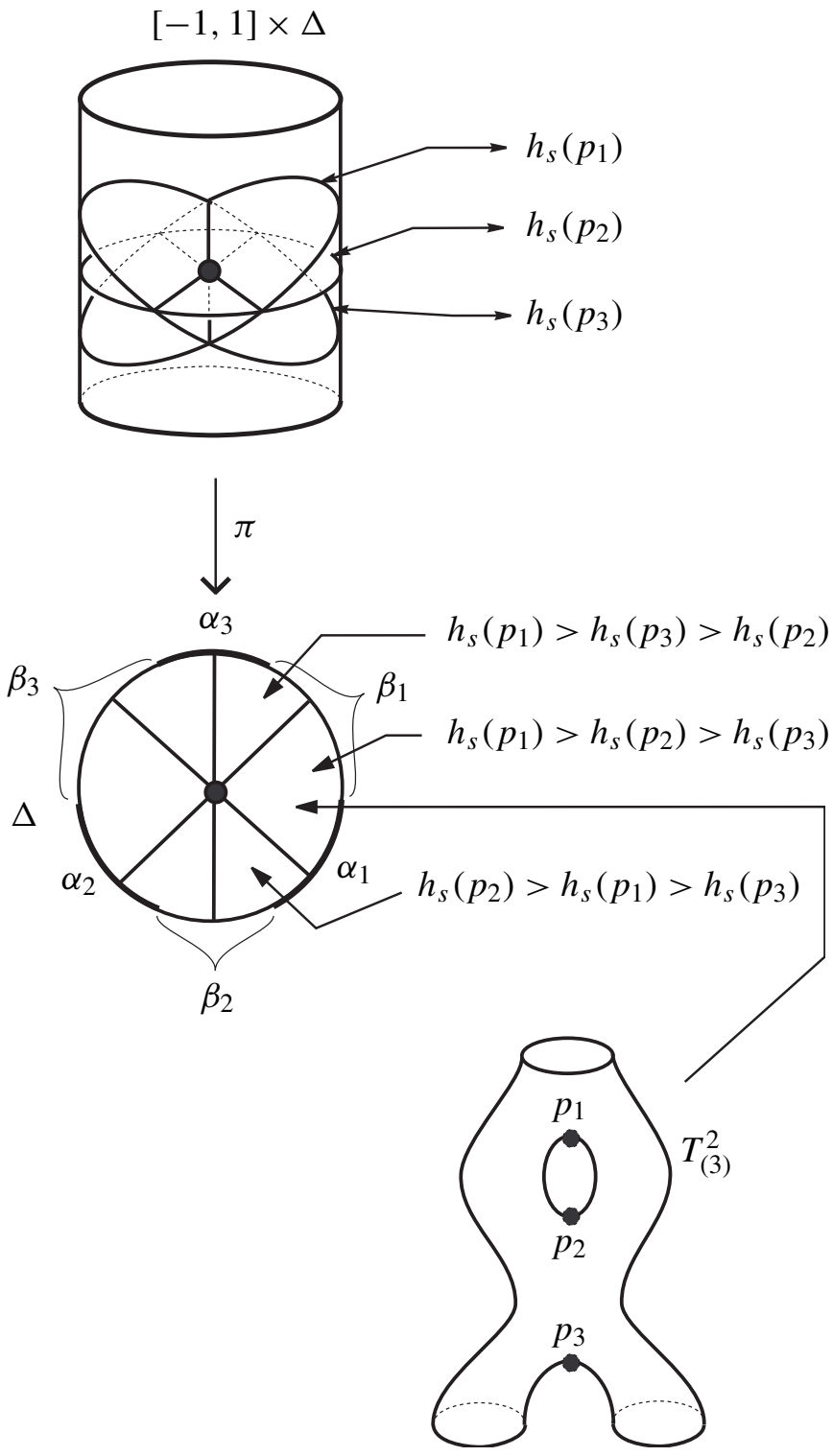

Figure 14. Family of functions corresponding to a singular fiber of type III $^{8}$.

over the middle point of $\alpha_{i}$ corresponds to the singular fiber of type $\mathrm{II}^{3}$. The compact 4-manifold $P_{i}$ is obtained from $(\pi \circ g)^{-1}\left(\alpha_{i}\right) \times[0,1]$ by attaching $D^{2}$ bundles, $D^{3}$-bundles and a 4-disk as in Section 4, where the last 4-disk corresponds to the $\mathrm{II}^{3}$-type singular fiber. 


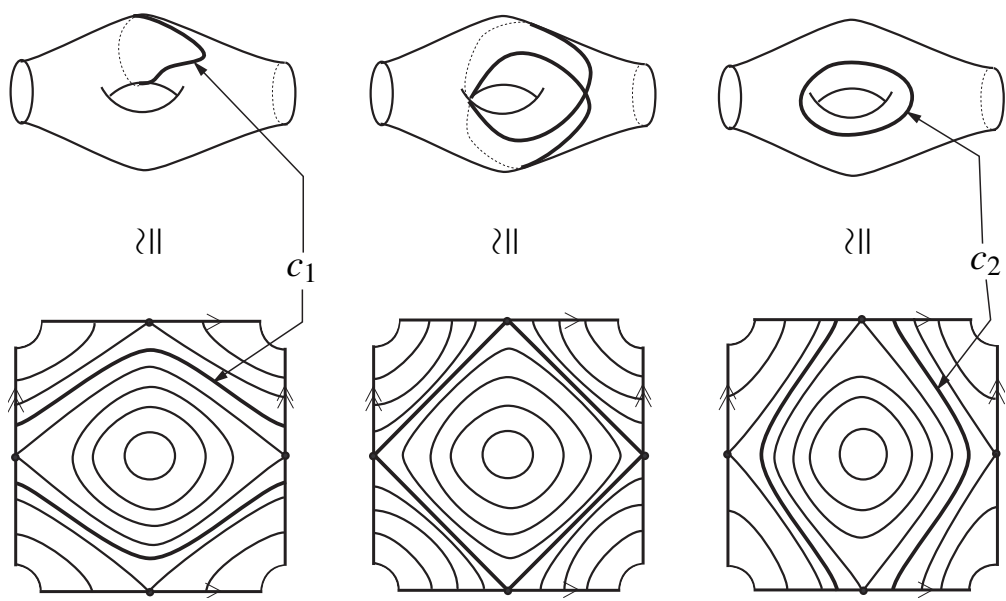

Figure 15. Family of functions corresponding to $\mathrm{II}^{3}$.

By attaching $D^{2}$-bundles over arcs and three copies of $D^{3}$ to each component of $(\pi \circ g)^{-1}\left(\partial \alpha_{i}\right)$, we get two copies of the solid torus. Hence $\partial P_{i}$ is diffeomorphic to the union of two solid tori attached along their boundaries. The attaching map sends the boundary $c_{1}$ of a meridian disk ${ }^{1}$ to a simple closed curve on the boundary of the other solid torus that intersects with the boundary $c_{2}$ of its meridian disk transversely at one point. For details, see Figure 15, which shows how the fibers change around a singular fiber of type $\mathrm{II}^{3}$. (Note that each component of a regular fiber bounds a disk by virtue of the construction of $V$.) Hence, $\partial P_{i}$ is diffeomorphic to $S^{3}$. Since $P_{i}$ is obtained from $\partial P_{i} \times[0,1]$ by attaching a 4-disk, we see that $P_{i}$ is diffeomorphic to $D^{4}$.

On the other hand, the piece $Q_{i}$ corresponding to the $\operatorname{arc} \beta_{i}$ for $i=1,2,3$ on $\partial \Delta$ as shown in Figure 14 is diffeomorphic to $\left(S^{1} \times D^{2}\right) \times[-1,1]$. This is because the singular fiber of type $\mathrm{II}^{3}$ over the middle point of $\beta_{i}$ corresponds to interchanging the heights of the bottom two critical points of the function $h_{s}: T_{(3)}^{2} \rightarrow[-1,1]$, where $s \in \beta_{i}$. In terms of the quotient space, its behavior is similar to what is depicted in Figure 7. Therefore, $Q_{i}$ is obtained from a 4-disk (corresponding to the $\mathrm{II}^{3}$-type singular fiber) by attaching $D^{3} \times \beta_{i}$ (corresponding to the top critical point of $\left.h_{s}\right)^{2}$ along $\left(D_{1}^{2} \cup D_{2}^{2}\right) \times \beta_{i}$, where $D_{1}^{2}$ and $D_{2}^{2}$ are disjoint 2-disks in $\partial D^{3}$. Therefore, $Q_{i}$ is diffeomorphic to $\left(S^{1} \times D^{2}\right) \times \beta_{i} \cong\left(S^{1} \times D^{2}\right) \times[-1,1]$, since over each end point of $\beta_{i}$ we have a solid torus, which is orientable.

Consequently, $F_{j}^{\prime \prime}$ is diffeomorphic to the compact 4-manifold obtained from three 4-disks $P_{1}, P_{2}$ and $P_{3}$ by attaching them appropriately along solid tori. Thus

\footnotetext{
${ }^{1} \mathrm{~A}$ properly embedded 2-disk in a solid torus is a meridian disk if its boundary is not null homotopic in the boundary torus.

${ }^{2}$ Each 3-disk $D^{3} \times\{*\}$ corresponds to that in the lower-left figure of Figure 10 .
} 
$P_{1}$ can be regarded as a 0 -handle and then $P_{2}$ is regarded as a 2-handle attached to $P_{1}$ along an unknotted circle on $\partial P_{1}$, since the exterior of the attaching circle in $\partial P_{1}$ is again a solid torus. Furthermore, $F_{j}^{\prime}$ is diffeomorphic to $T^{2} \times D^{2}$, and $P_{3} \cup F_{j}^{\prime}$ is diffeomorphic to $D^{4}$ since $P_{3} \cap F_{j}^{\prime}$ is diffeomorphic to $T^{2} \times \alpha_{3}$.

Therefore, $F_{j}$ is diffeomorphic to the closed 4-manifold consisting of the 0 handle $P_{1}$, the 2-handle $P_{2}$ attached to $P_{1}$ along an unknotted circle on $\partial P_{1}$, and a 4-handle. In particular, the boundary of $P_{1} \cup P_{2}$ must be diffeomorphic to $S^{3}$ so that the framing of the 2-handle $P_{2}$ must be equal to \pm 1 ; see [Kirby 1989], for example. Therefore, $F_{j}$ must be diffeomorphic to the complex projective plane $\mathbb{C} P^{2}$ up to orientation.

Remark 5.3. It is easy to see that the singular fibers appearing in Figure 9 can be embedded in the 2 -sphere, except for the $\mathrm{III}^{8}$-type singular fiber. This fact implies that the corresponding singular fiber is associated with a 2-parameter family of smooth functions on a punctured 2-sphere, as pointed out in the proof of Lemma 5.1. The III $^{8}$-type singular fiber cannot be embedded in the 2-sphere, but can be embedded in the 2-dimensional torus (see the proof of Lemma 5.2). The proofs above show that this fact is essential in distinguishing the $\mathrm{III}^{8}$-type singular fiber from the others.

We have proved that every closed oriented 4-manifold is oriented cobordant to the disjoint union of a finite number of copies of $\pm \mathbb{C} P^{2}$.

Let us consider the homomorphism $\varphi: \mathbb{Z} \rightarrow \Omega_{4}$ defined by $\varphi(1)=\left[\mathbb{C} P^{2}\right]$. This is a well-defined homomorphism, and is surjective by the argument above.

Let

$$
\sigma: \Omega_{4} \rightarrow \mathbb{Z}
$$

be the homomorphism defined by associating to each oriented cobordism class the signature of its representative. Classical techniques in algebraic topology show that this defines a well-defined homomorphism; for example, see [Thom 1952].

Since $\sigma\left(\left[\mathbb{C} P^{2}\right]\right)=1$, we see that the composition $\sigma \circ \varphi: \mathbb{Z} \rightarrow \mathbb{Z}$ is the identity. Therefore, $\varphi$ is injective. Thus, we get the following, which was originally proved by Rohlin [1952]; see also [Guillou and Marin 1986].

Theorem 5.4. The 4-dimensional oriented cobordism group $\Omega_{4}$ is infinite cyclic and is generated by the oriented cobordism class of $\mathbb{C} P^{2}$. In fact, the homomorphism (5-1) is an isomorphism.

Our proof shows that the complex projective plane naturally appears around each singular fiber of type $\mathrm{III}^{8}$, and therefore $\mathbb{C} P^{2}$ can be regarded as the genuinely natural representative of the generator of $\Omega_{4} \cong \mathbb{Z}$.

As a corollary to our proof, we get the following, which was originally obtained in [Saeki and Yamamoto 2006]. The proof given there is somewhat complicated 
since it depends on the classification of singular fibers of $C^{\infty}$ stable maps of $n$ dimensional manifolds into $(n-1)$-dimensional manifolds for $n \leq 5$, whereas our proof needs only the classification of such singular fibers for $n \leq 4$.

Corollary 5.5. Let $f$ be a $C^{\infty}$ stable map of a closed oriented 4-manifold $M$ into a 3-manifold $N$. Then the number of singular fibers of $f$ of type $\mathrm{III}^{8}$ counted with signs coincides with the signature of $M$.

The sign of a singular fiber of type $\mathrm{III}^{8}$ is +1 (or -1$)$ if the corresponding manifold $F_{j}$ is oriented diffeomorphic to $\mathbb{C} P^{2}$ (respectively $\overline{\mathbb{C} P^{2}}$ ). This sign convention must coincide with the one in [Saeki and Yamamoto 2006] since Corollary 5.5 determines the sign uniquely.

Gromov [2009] studied estimates for the number of self-intersections of the critical value set of a generic map from one manifold to another in terms of the topology of the source manifold. Corollary 5.5 gives a model case for such a study, as pointed out by Gromov.

Corollary 5.6. Let $f$ be a smooth map of a closed oriented n-dimensional manifold $M$ into an ( $n-1)$-dimensional manifold $N$ for $n \geq 4$, and suppose its singular fibers are (iterated suspensions of) those of $C^{\infty}$ stable maps of codimension $\leq 3$ not of type III $^{8}$ (that is, the singular fibers of $f$ are as in Figures 4 and 9 but without the III $^{8}$-type). Then the manifold $M$ is oriented null cobordant.

For the proof, we again need Kalmár's bundle structure theorem concerning the structure group since we need to deal with smooth fiber bundles with fiber $S^{4}$. Note that this corollary generalizes [Kalmár 2009, Corollary 2.7] about simple fold maps.

In particular, if $M$ is not oriented null cobordant, then every generic map $M \rightarrow N$ has a singular fiber of codimension $\geq 4$ or (an iterated suspension of) a singular fiber of type III $^{8}$.

Remark 5.7. Unfortunately, our technique in this paper does not directly apply for computing the 3 -dimensional unoriented cobordism group $\mathfrak{N}_{3}$. This is because the 2-dimensional one is not trivial, and we cannot fill $\mathbb{R} P^{2}$-bundles over arcs and circles. For similar reasons, our method cannot directly be used for computating $\mathfrak{N}_{m}$ for $m \geq 4$ and $\Omega_{n}$ for $n \geq 5$.

\section{Acknowledgments}

I would like to express my sincere gratitude to Boldizsár Kalmár and Takahiro Yamamoto for stimulating discussions and invaluable comments. I also thank the referee for helpful comments that improved the presentation of the paper. 


\section{References}

[Costantino and Thurston 2008] F. Costantino and D. Thurston, "3-manifolds efficiently bound 4manifolds", J. Topol. 1:3 (2008), 703-745. MR 2009g:57034 Zbl 1166.57016

[Golubitsky and Guillemin 1973] M. Golubitsky and V. Guillemin, Stable mappings and their singularities, Graduate Texts in Math. 14, Springer, New York, 1973. MR 49 \#6269 Zbl 0294.58004

[Gromov 2009] M. Gromov, "Singularities, expanders and topology of maps, I: Homology versus volume in the spaces of cycles", Geom. Funct. Anal. 19:3 (2009), 743-841. MR 2563769 Zbl 05652310

[Guillou and Marin 1986] L. Guillou and A. Marin (editors), À la recherche de la topologie perdue, Progress in Mathematics 62, Birkhäuser, Boston, 1986.

[Hatcher 1983] A. E. Hatcher, "A proof of the Smale conjecture, $\operatorname{Diff}\left(S^{3}\right) \simeq \mathrm{O}(4)$ ", Ann. of Math. (2) 117:3 (1983), 553-607. MR 85c:57008 Zbl 0531.57028

[Hiratuka 2001] J. T. Hiratuka, A fatorização de Stein e o número de singularidades de aplicações estáveis, thesis, University of São Paulo, 2001.

[Kalmár 2008] B. Kalmár, "Pontryagin-Thom-Szúcs type construction for non-positive codimensional singular maps with prescribed singular fibers", pp. 66-79 in The second Japanese-Australian Workshop on Real and Complex Singularities, RIMS Kôkyûroku 1610, Research Institute for Mathematical Sciences, Kyoto University, 2008.

[Kalmár 2009] B. Kalmár, "Fold maps and immersions from the viewpoint of cobordism", Pacific J. Math. 239:2 (2009), 317-342. MR 2009i:57063 Zbl 1171.57028

[Kirby 1989] R. C. Kirby, The topology of 4-manifolds, Lecture Notes in Math. 1374, Springer, Berlin, 1989. MR 90j:57012 Zbl 0668.57001

[Kushner et al. 1984] L. Kushner, H. Levine, and P. Porto, "Mapping three-manifolds into the plane, I", Bol. Soc. Mat. Mexicana (2) 29:1 (1984), 11-33. MR 86j:58011 Zbl 0586.57018

[Levine 1985] H. Levine, Classifying immersions into $\mathbb{R}^{4}$ over stable maps of 3-manifolds into $\mathbb{R}^{2}$, Lecture Notes in Math. 1157, Springer, Berlin, 1985. MR 88f:57056 Zbl 0567.57001

[Mather 1971] J. N. Mather, "Stability of $C^{\infty}$ mappings, VI: The nice dimensions", pp. 207-253 in Singularities Symposium (Liverpool, 1969/70), vol. 1, Lecture Notes in Math. 192, Springer, Berlin, 1971. MR 45 \#2747

[Milnor and Stasheff 1974] J. W. Milnor and J. D. Stasheff, Characteristic classes, Annals of Mathematics Studies 76, Princeton University Press, 1974. MR 55 \#13428 Zbl 0298.57008

[Pontryagin 1955] L. S. Pontryagin, "Smooth manifolds and their applications in homotopy theory", Trudy Mat. Inst. im. Steklov. 45, Izdat. Akad. Nauk SSSR, Moscow, 1955. In Russian; translated in pp. 1-114 of American Mathematical Society Translations (2) 11, American Mathematical Society, Providence, RI, 1959. MR 22 \#5980 Zbl 0064.17402

[Rohlin 1951] V. A. Rohlin, "A three-dimensional manifold is the boundary of a four-dimensional one”, Doklady Akad. Nauk SSSR (N.S.) 81 (1951), 355-357. In Russian. MR 14,72g

[Rohlin 1952] V. A. Rohlin, "New results in the theory of four-dimensional manifolds", Doklady Akad. Nauk SSSR (N.S.) 84 (1952), 221-224. In Russian. MR 14,573b

[Saeki 2004] O. Saeki, Topology of singular fibers of differentiable maps, Lecture Notes in Mathematics 1854, Springer, Berlin, 2004. MR 2005m:58085 Zbl 1072.57023

[Saeki and Yamamoto 2006] O. Saeki and T. Yamamoto, "Singular fibers of stable maps and signatures of 4-manifolds", Geom. Topol. 10 (2006), 359-399. MR 2007f:57058 Zbl 1107.57019

[Smale 1959] S. Smale, "Diffeomorphisms of the 2-sphere", Proc. Amer. Math. Soc. 10 (1959), 621-626. MR 22 \#3004 Zbl 0118.39103 
[Thom 1952] R. Thom, Quelques propriétés des variétés-bords, Colloque de Topologie de Strasbourg 1951 5, La Bibliothèque Nationale et Universitaire de Strasbourg, 1952. MR 14,492a

[Thom 1954] R. Thom, "Quelques propriétés globales des variétés différentiables", Comment. Math. Helv. 28 (1954), 17-86. MR 15,890a Zbl 0057.15502

[Wall 1959] C. T. C. Wall, "Note on the cobordism ring", Bull. Amer. Math. Soc. 65 (1959), 329-331. MR 21 \#6586 Zbl 0128.16801

[Yamamoto 2006] T. Yamamoto, "Classification of singular fibres of stable maps of 4-manifolds into 3-manifolds and its applications”, J. Math. Soc. Japan 58:3 (2006), 721-742. MR 2007m:57035 Zbl 1105.57027

[Yamamoto 2007] T. Yamamoto, "Euler number formulas in terms of singular fibers of stable maps", pp. 427-457 in Real and complex singularities (Sydney, 2005), edited by Y. Paunescu et al., World Sci. Publ., Hackensack, NJ, 2007. MR 2008m:57071 Zbl 1143.57016

Received August 2, 2009. Revised October 19, 2009.

OSAMU SAEKI

FACULTY OF MATHEMATICS

KYUSHU UNIVERSITY

MoTOOKA 744, NisHI-KU

FUKUOKA 819-0395

JAPAN

saeki@math.kyushu-u.ac.jp

http://www2.math.kyushu-u.ac.jp/ saeki/index.html 


\title{
PACIFIC JOURNAL OF MATHEMATICS
}

\author{
http://www.pjmath.org \\ Founded in 1951 by \\ E. F. Beckenbach (1906-1982) and F. Wolf (1904-1989)
}

\section{EDITORS}

V. S. Varadarajan (Managing Editor)

Department of Mathematics

University of California

Los Angeles, CA 90095-1555

pacific@math.ucla.edu

Vyjayanthi Chari

Department of Mathematics

University of California

Riverside, CA 92521-0135

chari@math.ucr.edu

Robert Finn

Department of Mathematics Stanford University

Stanford, CA 94305-2125

finn@math.stanford.edu

Kefeng Liu

Department of Mathematics

University of California

Los Angeles, CA 90095-1555

liu@math.ucla.edu
Darren Long

Department of Mathematics

University of California

Santa Barbara, CA 93106-3080

long@math.ucsb.edu

Jiang-Hua Lu

Department of Mathematics

The University of Hong Kong

Pokfulam Rd., Hong Kong jhlu@maths.hku.hk

Alexander Merkurjev

Department of Mathematics

University of California

Los Angeles, CA 90095-1555

merkurev@math.ucla.edu
Sorin Popa

Department of Mathematics University of California

Los Angeles, CA 90095-1555 popa@math.ucla.edu

Jie Qing

Department of Mathematics

University of California

Santa Cruz, CA 95064

qing@cats.ucsc.edu

Jonathan Rogawski

Department of Mathematics

University of California

Los Angeles, CA 90095-1555

jonr@math.ucla.edu

\section{PRODUCTION}

pacific@math.berkeley.edu

\begin{abstract}
Silvio Levy, Scientific Editor Matthew Cargo, Senior Production Editor
\end{abstract}
ACADEMIA SINICA, TAIPEI

CALIFORNIA INST. OF TECHNOLOGY

INST. DE MATEMÁTICA PURA E APLICADA

KEIO UNIVERSITY

MATH. SCIENCES RESEARCH INSTITUTE

NEW MEXICO STATE UNIV.

OREGON STATE UNIV.

\section{SUPPORTING INSTITUTIONS}

STANFORD UNIVERSITY
UNIV. OF BRITISH COLUMBIA
UNIV. OF CALIFORNIA, BERKELEY
UNIV. OF CALIFORNIA, DAVIS
UNIV. OF CALIFORNIA, LOS ANGELES
UNIV. OF CALIFORNIA, RIVERSIDE
UNIV. OF CALIFORNIA, SAN DIEGO
UNIV. OF CALIF., SANTA BARBARA

UNIV. OF CALIF., SANTA CRUZ

UNIV. OF MONTANA

UNIV. OF OREGON

UNIV. OF SOUTHERN CALIFORNIA

UNIV. OF UTAH

UNIV. OF WASHINGTON

WASHINGTON STATE UNIVERSITY

These supporting institutions contribute to the cost of publication of this Journal, but they are not owners or publishers and have no responsibility for its contents or policies.

See inside back cover or www.pjmath.org for submission instructions.

The subscription price for 2010 is US \$420/year for the electronic version, and \$485/year for print and electronic.

Subscriptions, requests for back issues from the last three years and changes of subscribers address should be sent to Pacific Journal of Mathematics, P.O. Box 4163, Berkeley, CA 94704-0163, U.S.A. Prior back issues are obtainable from Periodicals Service Company, 11 Main Street, Germantown, NY 12526-5635. The Pacific Journal of Mathematics is indexed by Mathematical Reviews, Zentralblatt MATH, PASCAL CNRS Index, Referativnyi Zhurnal, Current Mathematical Publications and the Science Citation Index.

The Pacific Journal of Mathematics (ISSN 0030-8730) at the University of California, c/o Department of Mathematics, 969 Evans Hall, Berkeley, CA 94720-3840, is published monthly except July and August. Periodical rate postage paid at Berkeley, CA 94704, and additional mailing offices. POSTMASTER: send address changes to Pacific Journal of Mathematics, P.O. Box 4163, Berkeley, CA 94704-0163.

PJM peer review and production are managed by EditFLOW ${ }^{\mathrm{TM}}$ from Mathematical Sciences Publishers.

PUBLISHED BY PACIFIC JOURNAL OF MATHEMATICS

at the University of California, Berkeley 94720-3840

A NON-PROFIT CORPORATION

Typeset in LATEX

Copyright $(\mathrm{C} 2010$ by Pacific Journal of Mathematics 


\section{PACIFIC JOURNAL OF MATHEMATICS}

Volume $248 \quad$ No. $1 \quad$ November 2010

An existence theorem of conformal scalar-flat metrics on manifolds with boundary 1

SÉRgio de Moura Almaraz

Parasurface groups

KHALID BOU-RABEE

Expressions for Catalan Kronecker products

Andrew A. H. Brown, Stephanie Van Willigenburg and Mike

ZABROCKI

Metric properties of higher-dimensional Thompson's groups

José BURILLO and SEAN CLEARY

Solitary waves for the Hartree equation with a slowly varying potential

KIRIL DATCHEV and IVAN VENTURA

Uniquely presented finitely generated commutative monoids

PEDRO A. GARCíA-SÁNCHEZ and IgnaCio OJEDA

The unitary dual of $p$-adic $\widetilde{\operatorname{Sp}(2)}$

MARCELA HANZER and IVAN MATIĆ

A Casson-Lin type invariant for links

ERIC HARPER and NIKOLAI SAVELIEV

Semiquandles and flat virtual knots

ALLISON HENRICH and SAM NELSON

Infinitesimal rigidity of polyhedra with vertices in convex position

IVAN IZMESTIEV and JEAN-MARC SCHLENKER

Robust four-manifolds and robust embeddings

VYACHESLAV S. KRUSHKAL

On sections of genus two Lefschetz fibrations

SINEM ÇELIK ONARAN

Biharmonic hypersurfaces in Riemannian manifolds

YE-LIN OU

Singular fibers and 4-dimensional cobordism group 